\title{
Correction Factors for the Analysis of Voltammetric Peak Currents Measured using Staircase Voltammetry
}

\author{
Christopher Batchelor-McAuley ${ }^{1}$, Minjun Yang $^{1}$, Elise M. Hall ${ }^{1}$, Richard G. Compton ${ }^{1}$ \\ ${ }^{1}$ Department of Chemistry, Physical and Theoretical Chemistry Laboratory, University of Oxford, South Parks Road, Oxford OX1 \\ 3QZ, United Kingdom
}

\begin{abstract}
The use of staircase voltammetry for the measurement of reversible diffusional redox species under a linear mass-transport regime leads to significant suppression of the peak currents (up to $20 \%$ for larger step sizes) and an increase in the peak-to-peak separation (cf. $\Delta \mathbf{E}_{\mathrm{pp}} \sim 70 \mathrm{mV}$ at the reversible limit as opposed to $57 \mathrm{mV}$ ). These discrepancies between the voltammetric results of a staircase and true analog voltammogram may lead to mis-interpretation of data. This work provides an over view of the differences between the two techniques in the macro-electrode reversible limit and provides new expressions which allow the peak current of a staircase voltammogram to be quantitatively analysed. Moreover, clear guidance is provided about which conditions the application of the provided equation is valid in the terms of mass-transport edge effects to the macro-electrode.
\end{abstract}

\section{KEYWORDS}

Electrochemistry, Diffusion Coefficient, Electron Transfer Kinetics, Randles-Ševčík Equation

Address correspondence to Richard G. Compton, Richard.compton@chem.ox.ac.uk 


\section{Introduction}

The analysis of cyclic voltammograms either by the use of analytical expressions[1] or numerical simulation[2-4] almost invariably assumes that the applied potential is a true linear ramp i.e. $\mathrm{dE} / \mathrm{dt}$ is taken to be a constant. For the majority of modern commercial potentiostats available today the basic cyclic voltammetric method available with the equipment in fact applies a staircase ramping potential. Commonly, the assumption is that for sufficiently small step sizes then the results of the two methodologies converge. At a qualitative level this assumption is often reasonably valid but as will be discussed within this work, for the measurement of physically correct experimental parameters and the extraction of quantitative data, insight into the differences between these two voltammetric methodologies is required.

From the mid-80s till the early 90s Osteryoung, amongst others, [5,6] published a series of important papers focused on the discrepancies between staircase and true linear sweep voltammetry.[7-12] As with many pulse techniques[13] the initial motivation for interest in the application of staircase voltammetry was in order to facilitate and improve the measurement of Faradaic processes over capacitative.[14] This improved resolution between the two current sources may be achieved for diffusional species by exploiting the difference in the currents' time constants. Crudely the current associated with the capacitative charging of the electrode varies exponentially with time $\left(\mathrm{I}=f\left(\mathrm{e}^{-\mathrm{kt}}\right)\right)$ whereas for a diffusion limited process (under a linear diffusion regime) the current varies with the square-root of time ( $\mathrm{I}=$ $f\left(\mathrm{t}^{-0.5}\right)$ ). In running a staircase voltammogram the potentiostat is in effect performing a series of short sequential chronoamperograms. Figure 1 depicts a comparison of the voltage waveforms used by the two different voltammetric techniques. For the staircase waveform (red) the point at which the current is sampled during the course of each step influecnes the shape of the voltammetric response. By convention the sampling position is define by the dimensionless value alpha, where an alpha of one correcsponds to sampling the current at the end of a potenital step and conversly an alpha of zero corresponds to sampling at the start. It is worth noting that this sampling alpha value should not be conflated with the transfer coefficient 'alpha' used within the Butler-Volmer equation. 


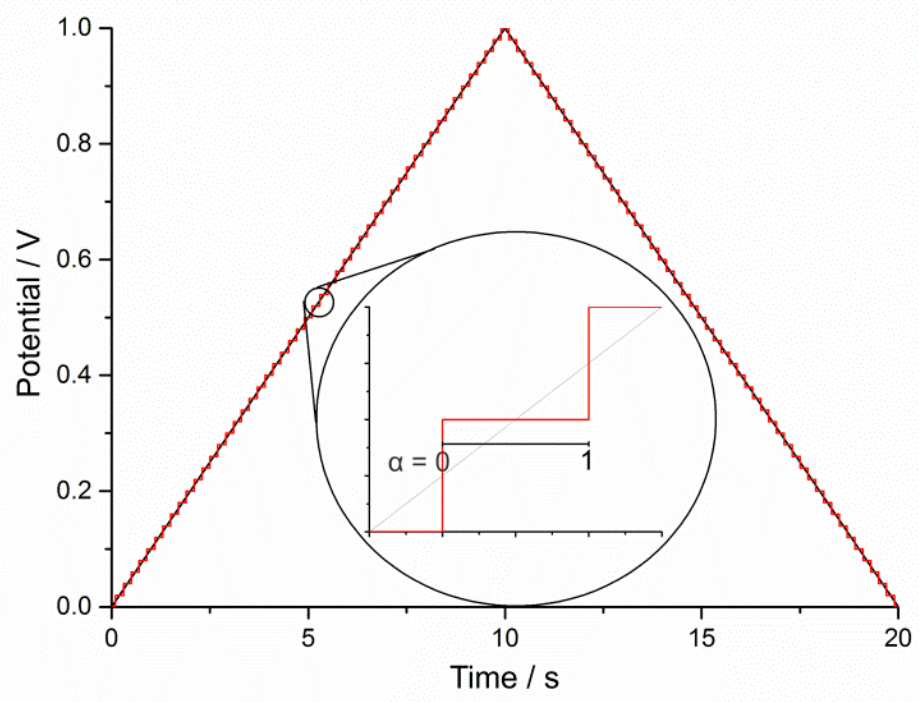

Figure 1: Comparison of the voltage wave forms used for staircase (red) and true analogue (black) cyclic voltammetry. Zoomed inlay depicts an individual step showing the sampling alpha scale, when $\alpha=1$ the current is sampled at the end of the step alternatively, $\alpha=0$ implies a current measurement at the beginning of the step. Data depicts the wave form used for a cyclic voltammogram $(0-1 \mathrm{~V})$ at a scan rate of $0.1 \mathrm{~V} \mathrm{~s}^{-1}$ and with a step potential of $20 \mathrm{mV}$. Image taken from ref 2.

As the potentiostat applies the staircase voltage ramp, the actual current passed at the electrode will exhibit sharp pulses associated with the application of each potential step. By sampling the current once during each potential step (as is commonly done), or alternatively averaging the recorded response, the large fluctuations in the current may be omitted and the current response will more closely resemble that found for conventional 'true' linear sweep voltammetry. For the majority of basic potentiostat procedures the current is sampled once at the end of the step (sampling alpha $=1.0$ ) hence due to their differing time constants the relative magnitude of the Faradaic current is greater than the capacitative current as compared to true analog cyclic voltammetry. However, although this method facilitates discrimination between diffusional Faradaic and capacitative currents under most conditions the resulting voltammogram is not equivalent to true linear sweep voltammetry as implemented using an analog potentiostat. For staircase voltammetry the procedure used for sampling the current significantly influence the resulting voltammogram and importantly leads to attenuated peak currents and increased peak-to-peak separations. These disparities between the recorded voltammograms will have certainly caused some researchers to conclude on a basis of their experimental data that a reversible redox couple is only quasi-reversible (or that the system 
exhibits ohmic distortions) and/or to under report the diffusion coefficient by ca. $20 \%$.

This work will focus upon the voltammetry of reversible diffusional redox couples and demonstrates both theoretically and experimentally the influence of the parameters used within staircase voltammetry. Consequently, correction factors for the Randles-Ševčík equation are provided, allowing quantitative analysis of voltammetric data of reversible species to be measured from staircase voltammetry without the direct need for simulation. Furthermore, this work also serves to clearly state the conditions under which a voltammetric system can be suitably and quantitatively analysed using these equations.

\section{Experimental}

Chemicals. Potassium chloride and hexaammineruthenium(III) chloride were supplied by Sigma-Aldrich. All solutions were made using ultrapure water from Millipore with resistivity not less than $18.2 \mathrm{M} \mathrm{cm}$ at $298 \mathrm{~K}$.

Electrochemistry. A three electrode system in a Faraday cage was used for all electrochemical experiments. A $\mu$ Autolab II from Metrohm Autolab B.V., Utrecht, The Netherlands was used as the potentiostat. The working electrode was a glassy carbon disk, BASi, (radius $=1.5 \mathrm{~mm})$, the electrode was polished on alumina $(1.0,0.3 \mu \mathrm{m}$, Buehler, Lake Bluff, IL, USA) and rinsed with deionised water thoroughly between each experiment. A saturated calomel electrode (SCE), BASi, was used as the reference and a graphite rod was used as the counter.

Simulations. The theoretical work in this paper utilises two models, first a one-dimensional system is provided where the electrode potential increases along a staircase ramp. Second, a two-dimensional model with an analog ramp is used to define the dimensionless scan rate at which the radial diffusion contributions to the current should be considered. The one dimension simulations use a script written in Python 2.7 whereas the two dimensional model was implemented in Julia,[15] a high performance scripting language. Further details of the models can be found within the SI section 1. 


\section{Results and discussion}

Figure 2 depicts the simulated response of a one-electron reversible redox couple at a macroelectrode (1D) to an applied staircase potential. The scan starts at $+0.2 \mathrm{~V}$ and the potential is reduced cathodically in increments of $5 \mathrm{mV}$. The current for ease of comparison has been normalized against the expected peak current for a reversible one-electron system. Unlike a regular voltammogram this staircase potential ramp results in significant pulsing of the current. At low overpotentials the magnitude of these current pulses are not significant, however as the formal potential of the redox couple is approached their magnitude increases. This increase in the magnitude of the pulsing simply reflects the change in the composition of the reactants and products at the interface as determined by the Nernst equation. As the potential is reduced further the depletion of the reactant at the electrode surface causes the reaction to become diffusion limited. Hence the redox response becomes less sensitive to the electrode potential and as such the magnitude of the pulsing decreases and the current tends

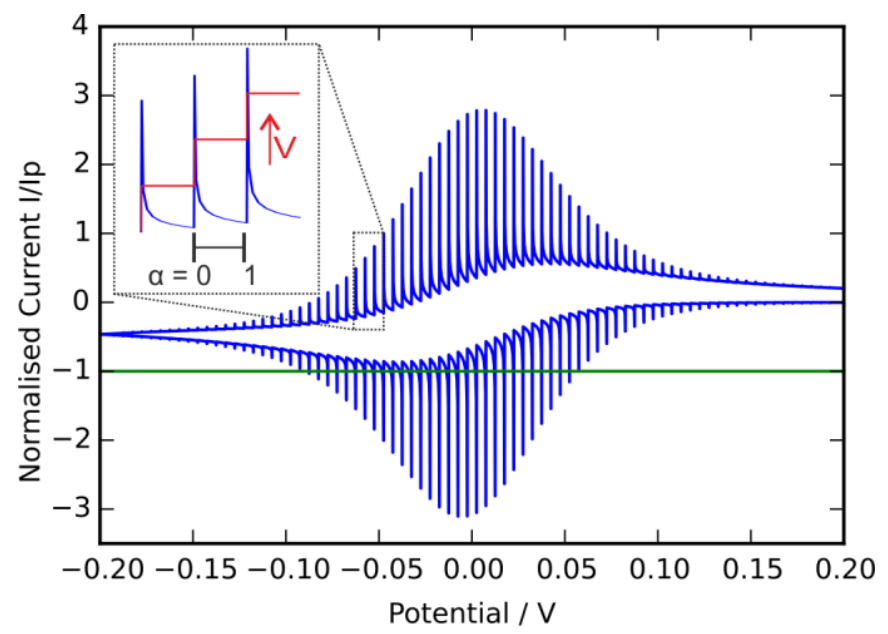

Figure 2: The voltammetric response of a reversible one-electron redox couple to a staircase potential ramp ( $5 \mathrm{mV}$ potential step, $\left.0.1 \mathrm{~V} \mathrm{~s}^{-1}\right)$ under a linear diffusion regime. Green line represents the expected current for an analog cyclic voltammogram. For ease of understanding the current has been plotted against the potential that would have been applied had an analog potential ramp been used. Inlay depicts a zoomed section of the voltammogram depicting three individual potential steps, showing both the variation of the potential (red line) and the scale used for the sampling value 'alpha'. Such that an alpha of one corresponds to a measurement at the end of a potential step and a value of zero corresponds to a measurement at the beginning of the step.

to its diffusion limited value. Upon reverse of the potential scan (at $-0.2 \mathrm{~V}$ ) the process is repeated in the anodic direction as the product is oxidised back to the starting material. The inlay depicts a zoomed region of the staircase voltammogram showing three individual steps. During the course of each step the current decreases, consequently depending upon how and when the current is sampled dramatically different voltammograms may be recorded. 
The convention for many modern potentiostats is to sample the current at the end of each potential step; this is reportedly to improve the resolution of the Faradaic signal above that of the capacitative background. However, as is clear from Figure 2, sampling the current at the end of the potential step will result in the recording of a current significantly less than would be attained if a true linear ramp was used. Figure 3 depicts the simulated results for the same (as shown in Figure 2) one-electron redox couple however here the current has only been sampled once per potential step. The position at which the current is sampled is defined by the dimensionless parameter 'alpha' (the inlay of Figure 1 schematically shows the scale used for the sampling alpha value). As shown in Figure 3 as the sampling position is altered the resulting voltammogram shifts from being larger than that expected for a simple analog linear sweep voltammogram to being smaller. A corresponding shift in the peak potentials are also observed as a function of the used sampling alpha value, where for an alpha of one the peakto-peak separation is significantly larger than that predicted for an analog voltammogram.

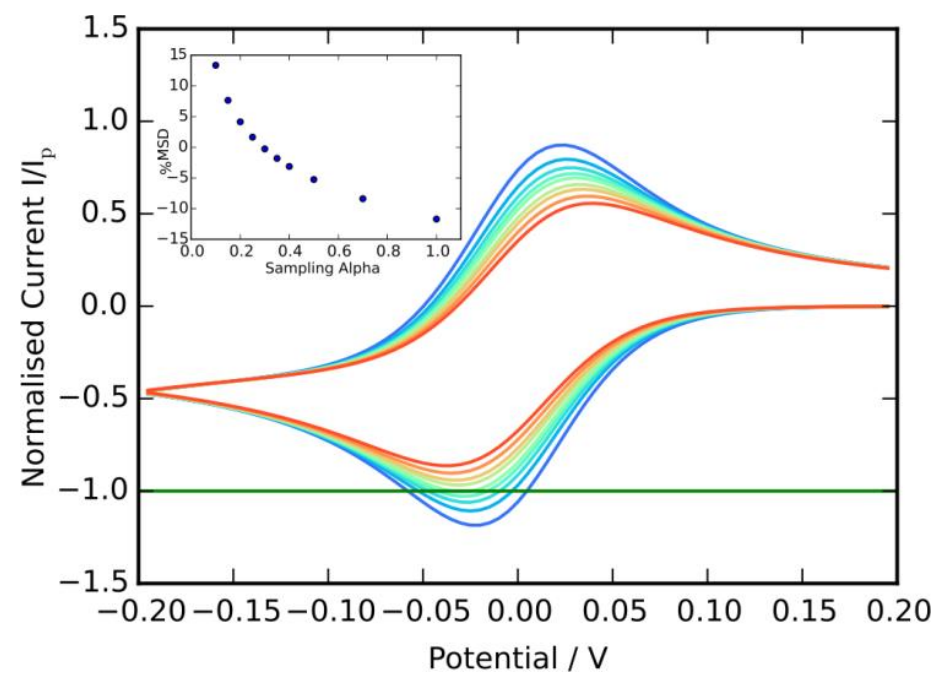

Figure 3: The simulated staircase cyclic voltammograms of a reversible oneelectron process where the current has been sampled once per potential step (5 $\left.m V, 0.1 \mathrm{~V} \mathrm{~s}^{-1}\right)$. The position of the sampling point is defined by the sampling parameter 'alpha' where an alpha of zero corresponds to sampling the current at the start of the potential step and a value of one for sampling at the end. Within the figure the sampling alpha value has been varied between 0.1 (blue line) to 1.0 (red line) [alpha $=0.1,0.15,0.2,0.25,0.3,0.35,0.4,0.5,0.7,1.0]$. Green line depicts the expected peak current for the one-electron reduction as predicted by the Randles-Ševčik equation. Inlay depicts the mean scaled deviation (\%MSD) of the simulated staircase voltammogram from that of ta 'true'linear sweep voltammogram as a function of the sampling alpha value.

Figure 3 allows assessment of the optimal 'alpha' value for the approximation of staircase voltammetry to that of true linear sweep voltammetry for a reversible one-electron diffusional redox species under a linear diffusion regime. From consideration of the peak current an 
alpha value of 0.3 is found to yield the closest correspondence between the two voltammetric techniques. In order to quantify the goodness of fit between the two voltammograms the mean scaled deviation (\%MSD) can be calculated, this value is defined here as:

$\% \mathrm{MSD}=\frac{1}{n} \sum_{n}\left(\frac{i_{\text {staircase }}-i_{\text {analog }}}{i_{\text {analog }}}\right) \times 100$

where $n$ is the number of data points, $i_{\text {staircase }}$ is the simulated current for the staircase voltammogram and $i_{\text {analog }}$ is the simulated current predicted for a voltamogram recorded using a true linear sweeping potential. The \%MSD value is calculated only using currents whose absolute magnitude is greater than $10 \%$ of the forward peak current.[16] This technique avoids weighting of the \%MSD value to low current regions. The inlay of Figure 3 plots the $\% \mathrm{MSD}$ of the staircase voltammograms and the 'true' linear sweep response as a function of the used sampling alpha value. From this it can be seen that using a sampling alpha value of 0.3 and a step potential of $5 \mathrm{mV}$ the \%MSD between the staircase voltammogram and the true analog linear ramp is a value of $-0.27 \%$ (see SI Section 2 for a direct comparison of the two voltammograms). The sign of this percentage value indicates if the staircase voltammogram is smaller (negative) or larger (positive) than the linear sweep equivalent. The magnitude of this \%MSD measurement will depend upon the step size used i.e. as the step size decreases the difference between the staircase and analog voltammetry is minismised. The following section serves to quantify both in terms of the peak current and the peak-topeak separation how the voltammetric shape is influenced by the step size.
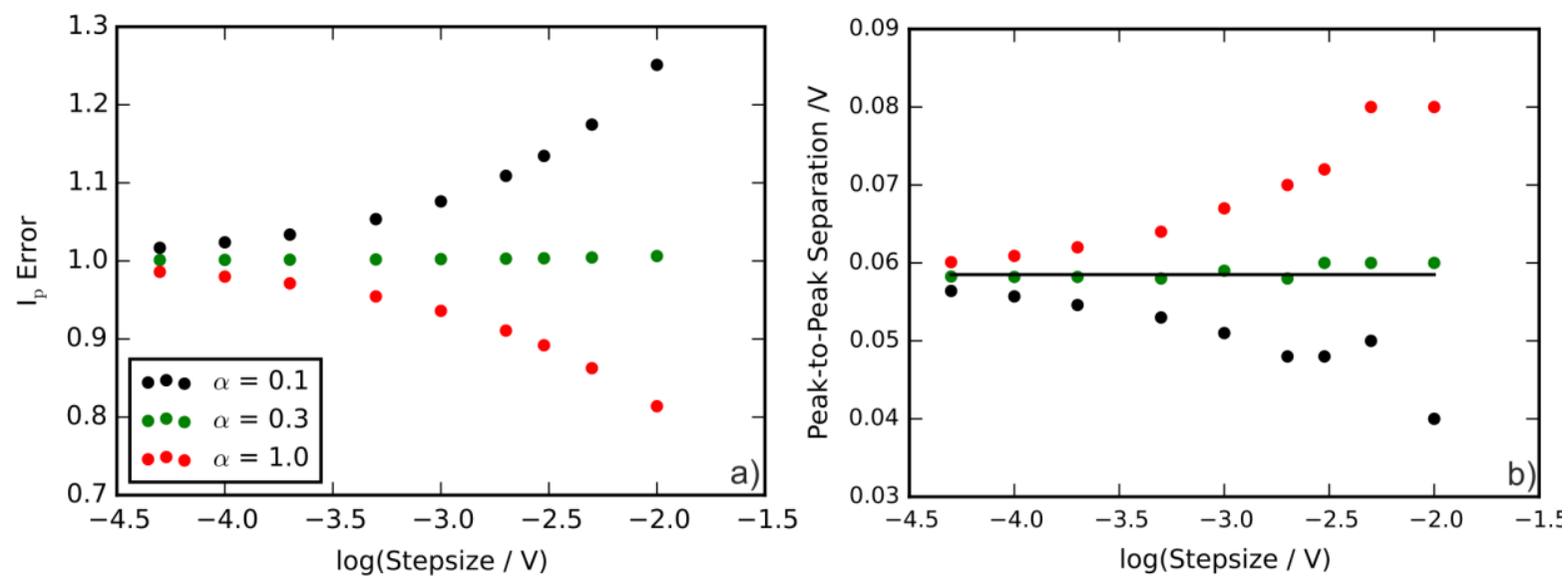

Figure 4: The variation of the peak current (a) and peak-to-peak separation (b) for staircase voltammetry as a function of step size (log base 10). For plot a) a value of unity for Ip error corresponds to the current expected for a true linear sweep voltammogram. From plot $b$ ) the solid line represents the expected peak-to-peak separation for a reversible redox couple using an analog potentiostat $(58.5 \mathrm{mV})$. The peak-to-peak separation is subject to a rounding error, such that, its magnitude can only be multiples of the step size, hence the slight irregularities observed for the trends in (b). 
Figure 4 a) plots the variation of the voltammetric peak current as a function of step size (values range from 10 to $0.05 \mathrm{mV}$ ), for a reversible electron transfer. The corresponding plot for an irreversible electron transfer process is shown in the SI Section 3 and demonstrates the lower sensitivity of the irreversible electron transfer to the use of staircase voltammetry. In Figure 4 a) a value of unity corresponds the peak current expected for a one electron redox species as given by the reversible form of the Randles-Ševčík equation:[17] [18]

$i_{p}=\left(2.69 \times 10^{5}\right) D^{0.5} C A n^{1.5} v^{0.5}$

where $i_{p}$ is the peak current (A), D is the diffusion coefficient $\left(\mathrm{m}^{2} \mathrm{~s}^{-1}\right), \mathrm{C}$ is the concentration of the analyte $\left(\mathrm{mol} \mathrm{m}^{-3}\right), \mathrm{A}$ is the area of the electrode $\left(\mathrm{m}^{2}\right)$ and $v$ is the experimental scan rate $\left(\mathrm{V} \mathrm{s}^{-1}\right)$. Even for 'relatively' small step sizes (cf. $1 \mathrm{mV}$ ), by sampling at the end of the potential step a significant error is incurred for peak current measurements. Consequently, direct application of the above Randles-Ševčík equation to voltammetric data measured using a staircase ramping potential will lead to erroneous results. It is commented that the use of step sizes below $\sim 0.2 \mathrm{mV}$ are required, for staircase voltammetry with an alpha value of one, to be quantitatively equivalent to true analog voltammetry ( $<3 \%$ error). However, for many commercial potentiostats the potential applied at the working electrode may be varied between $\pm 10 \mathrm{~V}$ (some potentiostats do allow smaller potential ranges to be utilized) where the digital-to-analog converter used is very commonly 16-bit. Consequently, the minimum usable step size is $0.305 \mathrm{mV}\left(=20 / 2^{16} \mathrm{~V}\right)$. Moreover, other factors relating to the potentiostat may render the use of such a 'small' step sizes impractical, hence it is not uncommon for step sizes of the magnitude of millivolts to be used by default. The error associated with the use of staircase voltammetry for measurements of diffusion coefficients even greater than the error of the magnitude of the due to the square root dependency of the peak current on the diffusion coefficient. Consequently, from the above it can be predicted that for an experimental step potential of $1 \mathrm{mV}$ the use of staircase voltammetry for the determination of the diffusion coefficient of a reversible one-electron redox couple will result in the under estimation of $\mathrm{D}$ by ca. $12.4 \%$ (for a step size of $5 \mathrm{mV}$ this leads to an underestimation by ca. 25.5\%). The size of this error is significantly larger than would be anticipated for all other sources of experimental error and represents a major discrepancy between the two voltammetric techniques. The variation of the peak-to-peak separation of the staircase 
voltammetry as a function of step size shows a similar trend, in that for larger step sizes greater deviations are observed. Note that for larger steps the peak-to-peak separation becomes subject to a rounding error. It is this rounding error that leads to the observed nonmonotonic variation of the peak-to-peak separation of the staircase voltammogram as a function of step size. As a final point it should be commented that the peak-to-peak separation, even for the analog potential ramp case, is sensitive to the turning potential of the cyclic voltammogram. Only when the turning potential is infinitely far past the peak potential of the redox couple is the ideal value of $57 / \mathrm{n} \mathrm{mV}$ approached.[1] The following section provides experimental verification of the above simulated results.

The diffusion coefficient of ruthenium(III) hexaamine in an aqueous solution with $0.1 \mathrm{M} \mathrm{KCl}$ supporting electrolyte is $8.43 \pm 0.03 \times 10^{-10} \mathrm{~m}^{2} \mathrm{~s}^{-1}$ (at $25^{\circ} \mathrm{C}$ ). [19] Figure 5 shows the oneelectron reduction of the ruthenium complex at a glassy carbon macroelectrode (radius $=1.5$ $\mathrm{mm}$ ) at a scan rate of $50 \mathrm{mV} \mathrm{s}^{-1}$, using a step potential of $2.44 \mathrm{mV}$ (the default size used by the potentiostat). The voltamograms have been recorded using two different sampling alpha values, 1.0 and 0.3. Also depicted is the peak current predicted by the Randles-Ševčík equation (Equation 2). In order to be explicitly clear about the value being referred to as the peak current, this measurement has been marked on the voltammogram. The peak current for

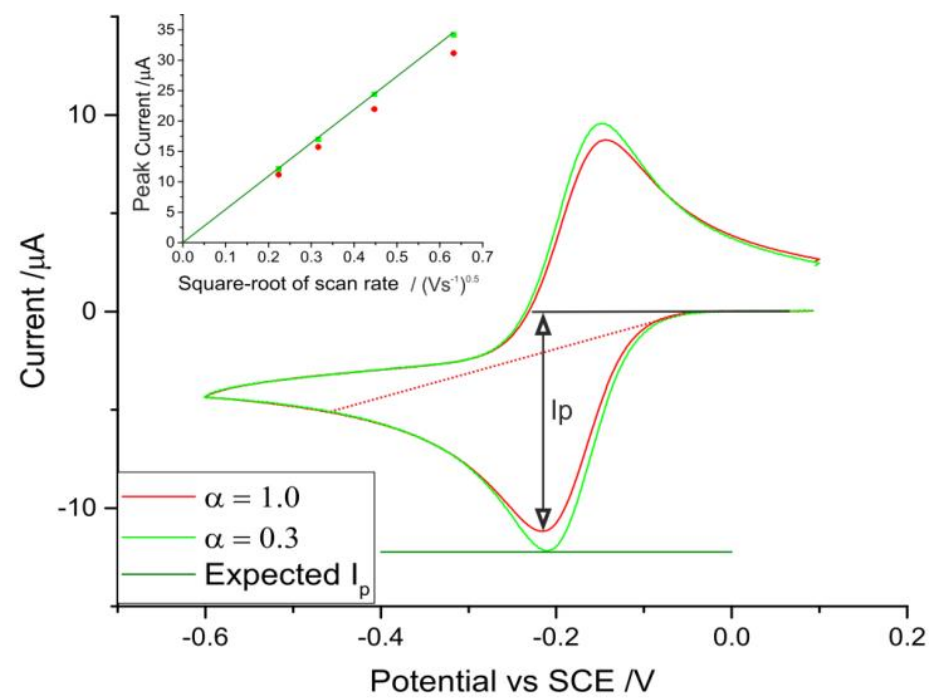

Figure 5: Experimentally recorded staircase voltammograms (step potential $2.44 \mathrm{mV}$ ) for the reduction of ruthenium(III) hexaamine $(0.1 \mathrm{M} \mathrm{KCl})$ at a glassy carbon electrode (radius $=1.5 \mathrm{~mm}$, baseline corrected). At a scan rate of 50 $m V s^{-1}$ and where the sampling alpha values of 0.3 and 1.0 have been used. Inlay depicts the variation of the peak currents as a function of the square root of the scan rate. Also depicted is an example of a correct baseline (black line) to be used for the measurement of a diffusional peak height. The red dashed line provides an example of a commonly selected but incorrect baseline. 
a diffusional redox species is measured relative to the extrapolated baseline as indicated and does not correspond to a line drawn from the onset of the wave to the diffusional tail (as shown by the red dashed line). Although the question of what is an appropriate baseline is dealt with in most books on voltammetry,[20,21] we have re-iterated this point here as it is not uncommon for 'automatic' peak selection methods available in various software packages to provide an incorrect baseline. Moreover, it is noted that even some potentiostat manuals demonstrate examples of assessment of diffusional voltammetric waves using incorrect background subtractions.

Figure 5 demonstrates how for staircase voltammetry by sampling at the end of the potential step the peak height is suppressed to $89.1 \%$ of the value expected for true linear sweep cyclic voltammetry and the peak-to-peak separation is increased from $61 \mathrm{mV}$ to $73 \mathrm{mV}$. These values are in excellent agreement with the above simulation which predicts a peak suppression of $90.1 \%$ and the respective peak-to-peak separations to be $58.5 \mathrm{mV}$ and $72 \mathrm{mV}$. Consequently if the Randles-Ševčík equation, as applicable to analog voltammetry, is used for the direct analysis of the voltammetric wave measured using staircase voltammetry with an alpha of 1.0 then the apparent diffusion coefficient of the ruthenium complex will be found to be 6.70 $\pm 0.03 \times 10^{-10} \mathrm{~m}^{2} \mathrm{~s}^{-1}$. This is less than the true value, as experimentally measured on a platinum microelectrode electrode by chronoamperometry, by $20.5 \%$. We comment that the use of a sampling alpha value of 0.3 yields a measured diffusion coefficient of $8.29 \pm 0.04 \times 10^{-10} \mathrm{~m}^{2} \mathrm{~s}^{-}$ 1 . This measured diffusion coefficient is within $2 \%$ of the literature value. Moreover, for this system using a sampling alpha value of 0.3 as opposed to 1.0 has not lead to a measurable change in the measured capacitative current. If a voltammogram of a reversible redox species has been measured on a macroelectrode using staircase voltammetry using an alpha value of 1.0 then care must be taken in analysing the data. To extract physically correct information from such systems either the voltammetric response can be simulated to explicitly account for the staircase potential or the analytical expressions used for data analysis needs to be modified to account for the differences between staircase and true linear sweep voltammetry. For instance using the above simulation a corrected form of the Randles-Ševčík can be provided to allow the use of the staircase votlammetric peak current to be quantitatively analysed. In combination with Table 1, which provides the appropriate correction factors (Cf), the expression below can be used to correct for the error incurred by using staircase voltammetry with a sampling alpha value of 1.0. 
In light of the above work it can be concluded that for a diffusional redox processes exhibiting reversible electron transfer kinetics the use of an alpha value of 0.3 yields a voltammogram that is a good approximation to that obtained by true analog voltammetry. Before concluding the article now turns to consider first, what conclusion may be erroneously drawn if an experimentalist is unaware of the differences between linear sweep and staircase voltammetry. Second, the brief consideration of other electrochemical systems will be broached.

Table 1: The correction factors for the peak current expression (Equation 3), for a reversible oneelectron process as a function of potential step size and using a sampling alpha value of 1.0.

\begin{tabular}{|l|l|}
\hline Step Size $/ \mathrm{mV}$ & Correction Factor (Cf) \\
\hline 0.05 & 0.986 \\
\hline 0.1 & 0.980 \\
\hline 0.2 & 0.971 \\
\hline 0.5 & 0.955 \\
\hline 1.0 & 0.936 \\
\hline 2.0 & 0.911 \\
\hline 2.44 & 0.901 \\
\hline 3 & 0.891 \\
\hline 5 & 0.863 \\
\hline 10 & 0.814 \\
\hline
\end{tabular}

Some work within the literature has suggested that the applicability of the Randles-Ševčík equation is only valid for a limited number of experimental cases.[22,23] However, it is plausible that this conclusion may have been influenced by the experimental voltammograms being recorded using staircase voltammetry. The distortion of the voltammetric wave i.e. the peak-height suppression compared to the diffusional tail and increased peak-to-peak separation may result in the conclusion that a voltammogram is distorted due to ohmic drop and/or increased mass-transport due to edge effects on the macroelectrode. On a macroelectrode it is commonly assumed that the diffusion to the electrode is purely linear, however for smaller electrodes or analytes with large diffusion coefficients the contribution to the Faradaic current arising from enhanced mass-transport to the electrode edge can lead to 
increased currents.[24] It is at this stage useful to quantify under which experimental conditions edge effects should be considered when analysing a voltammogram, this may be readily achieved through the use of a $2 \mathrm{D}$ simulation. For a disk electrode the transition from a linear to a radial diffusion regime is determined by the dimensionless scan rate. The dimensionless scan rate $(\sigma)$ can be defined as:

$\sigma=\frac{r^{2}}{D} \frac{F}{R T} v$

where $\mathrm{r}$ is the electrode radius (m), D is the diffusion coefficient of the analyte, $\mathrm{F}$ is the Faraday constant $\left(96485 \mathrm{C} \mathrm{mol}^{-1}\right), \mathrm{R}$ is the gas constant $\left(8.314 \mathrm{~J} \mathrm{~K}^{-1} \mathrm{~mol}^{-1}\right), \mathrm{T}$ is the temperature $(\mathrm{K})$ and $\mathrm{v}$ is the experimental scan rate. Consequently, from simulation it can be determined that when $\sigma$ is $>3350$, the influence of radial diffusion upon the peak current should lead to a peak that is no more than $3 \%$ greater than that expected from the RandlesŠevčík equation. For the above experimental system of the reduction of ruthenium(III) this means that edge effects upon the voltammogram only need to be considered when scan rates below $32 \mathrm{mV} \mathrm{s}^{-1}$ are used. The question of the presence of ohmic drop is more complex, such voltammetric distortions can arise from a variety of sources. However, in many experimental cases when the voltammogram is observed to be distorted this is commonly associated with the use of an inadequate concentration of supporting electrolyte. It is not irregular for the supporting electrolyte to analyte concentration ratio needed for ohmic distortions to be nonmeasurable to be greater than 100.[25] Two important comments should be appended here. First, the required support ratio varies as a function of the dimensionless scan rate. Second, the support ratio is sensitive to the diffusion coefficient of the analyte relative to that of the supporting electrolyte ions. Note the above experimental work on ruthenium(III) uses a support to analyte ratio of 100 and the diffusion coefficient of ruthenium complex is approximately half of that of the supporting electrolyte $(\mathrm{KCl})$ ions. Consequently, the above measured peak-to-peak separation of $61 \mathrm{mV}$ as opposed to the predicted value of $58.5 \mathrm{mV}$ (for the staircase voltammetry with a sampling alpha of 0.3 ) is completely consistent with being due to a minor influence of migration upon the voltammetric response. Other sources of ohmic distortion of a voltammetric response may be observed, however further discussion of the origins of such non-idealities is beyond the scope of this work. However, we comment that with appropriate correction voltammograms have been successfully recorded into the mega volts per second range.[26] As a final comment it is highlighted that both of the above 
two issues i.e. edge effects and migration are significantly more pronounced for species with high diffusion coefficients. For instance care must be taken when studying the reduction of protons,[27] the high diffusion coefficient (cf. $9.3 \times 10^{-9} \mathrm{~m}^{2} \mathrm{~s}^{-1}$ ) can and regularly does lead to complications with the voltammetry.

All of the above has only considered the situation in which an electrochemical reversible solution phase redox species is being studied, where it is concluded that using a sampling alpha value of 0.3 provides the best approximation to analog voltammetry. The benefit of the voltammogram being effectively equivalent to that of linear sweep voltammetry lies in the fact that the vast majority of simulations and analytical expressions are predicated upon the application of a true linear ramping potential, hence these well documented procedures can be used without modification. However, depending on the electrochemical system being studied the discrepancies between the two techniques may be more or less apparent, the important factor being how rapidly the current changes as a function of time. For instance under steadystate mass-transport conditions the voltammetric response will be far less sensitive to the form of the potential ramp used for measurement.[28] Conversely, for systems involving surface species i.e redox modified electrodes, underpotential deposition or stripping voltammetry,[8] huge differences between the voltammetric responses may be observed to the point that for a reversible redox process the voltammetric response may be completely unobservable via staircase voltammetry. On this note the interested reader is referred to the work of Heering et al. for further insights in to the complexities of the surface bound problem.[29]

\section{Conclusions}

The basic voltammetric technique provided by many modern potentiostats involves the application of a staircase ramping potential. The resulting voltammogram is in many cases qualitatively similar to that of a true analog sweeping potential however as demonstrated quantitative analysis can and does lead to significant errors in the reported values both in terms of the peak-to-peak separations and the peak current. By sampling the current not at the end of the potential step but at $30 \%$ along the steps total duration the resulting voltammogram is for even relatively large step sizes (ca. $10 \mathrm{mV}$ ) virtually equivalent to that recorded using a linear potential ramp. Although the potentiostat manuals highlight that there are differences between the two techniques, the vast majority of the procedures available for analysis of the resulting voltammograms do not account for the influence of the potential step. This work 
provides a modified form of the Randles-Ševčík equation which may be used to allow a more physically correct interpretation of the peak current for voltammograms of reversible species measured at a macro-electrode using a staircase ramping potential. Finally, the article considers under what experimental conditions the Randles-Ševčík equation is applicable; importantly the dimensionless scan rate needs to be greater than 3350 in order for the influence of radial diffusion to the electrode to be negligible.

\section{Acknowledgements}

The research leading to these results has received partial funding from the European Research Council under the European Union's Seventh Framework Programme (FP/2007-2013) / ERC Grant Agreement n. [320403]. 


\section{References}

[1] Nicholson, R. S.; Shain, I. Analytical Chemistry 1964, 36, 706-723.

[2] Batchelor-McAuley, C.; Kätelhön, E.; Barnes, E. O.; Compton, R. G.; Laborda, E.; Molina, A. ChemistryOpen 2015, 4, 224-260.

[3] Rudolph, M. Journal of Electroanalytical Chemistry 1994, 375, 89-99.

[4] Rudolph, M.; Reddy, D. P.; Feldberg, S. W. Analytical Chemistry 1994, 66, 589A-600A.

[5] Bott, A. W. Current Separations 1997, 16, 23-26.

[6] Miaw, L. H. L.; Boudreau, P. A.; Pichler, M. A.; Perone, S. P. Analytical Chemistry 1978, 50, 1988-1996.

[7] Bilewicz, R.; Osteryoung, R. A.; Osteryoung, J. Analytical Chemistry 1986, 58, 2761-2765.

[8] Bilewicz, R.; Wikiel, K.; Osteryoung, R.; Osteryoung, J. Analytical Chemistry 1989, 61, 965-972.

[9] Murphy, M. M.; O'Dea, J. J.; Arn, D.; Osteryoung, J. G. Analytical Chemistry 1989, 61, 2249-2254.

[10] Murphy, M. M.; O'Dea, J. J.; Arn, D.; Osteryoung, J. G. Analytical Chemistry 1990, 62, 903-909.

[11] Seralathan, M.; Osteryoung, R.; Osteryoung, J. Journal of Electroanalytical Chemistry 1986, 214, 141-156.

[12] Seralathan, M.; Osteryoung, R. A.; Osteryoung, J. G. Journal of Electroanalytical Chemistry 1987, 222, 69100 .

[13] Barker, G. C. Analytica Chimica Acta 1958, 18, 118-131.

[14] Christie, J. H.; Lingane, P. J. Journal of Electroanalytical Chemistry 1965, 10, 176-182.

[15] Bezanson, J.; Edelman, A.; Karpinski, S.; Shah, V. B. CoRR 2014, abs/1411.1607.

[16] Henstridge, M. C.; Laborda, E.; Wang, Y.; Suwatchara, D.; Rees, N.; Molina, A.; Martínez-Ortiz, F.; Compton, R. G. Journal of Electroanalytical Chemistry 2012, 672, 45-52.

[17] Randles, J. E. B. Transactions of the Faraday Society 1948, 44, 327-338.

[18] Ševčík, A. Collect. Czech. Chem. Commun. 1948, 13, 349-377.

[19] Wang, Y.; Limon-Petersen, J. G.; Compton, R. G. Journal of Electroanalytical Chemistry 2011, 652, 13-17.

[20] Compton, R. G.; Banks, C. E. Understanding Voltammetry; Imperial College Press, 2011.

[21] Bard, A. J.; Faulkner, L. R. Electrochemical Methods: Fundamentals and Applications; Wiley, 2000.

[22] Bentley, C. L.; Bond, A. M.; Hollenkamp, A. F.; Mahon, P. J.; Zhang, J. Analytical Chemistry 2013, 85, 2239-2245.

[23] Bentley, C. L.; Bond, A. M.; Hollenkamp, A. F.; Mahon, P. J.; Zhang, J. Analytical Chemistry 2014, 86, 2073-2081.

[24] Ngamchuea, K.; Eloul, S.; Tschulik, K.; Compton, R. G. Journal of Solid State Electrochemistry 2014, 18, 3251-3257.

[25] Dickinson, E. J. F.; Limon-Petersen, J. G.; Rees, N. V.; Compton, R. G. The Journal of Physical Chemistry C 2009, 113, 11157-11171.

[26] Amatore, C.; Maisonhaute, E.; Simonneau, G. Electrochemistry Communications 2000, 2, 81-84.

[27] Kätelhön, E.; Batchelor-McAuley, C.; Compton, R. G. The Journal of Physical Chemistry C 2015, In Press.

[28] Barnes, A. S.; Streeter, I.; Compton, R. G. Journal of Electroanalytical Chemistry 2008, 623, 129-133.

[29] Heering, H. A.; Mondal, M. S.; Armstrong, F. A. Analytical Chemistry 1999, 71, 174-182. 